\title{
A Case-Based Approach to Nosocomial Infection Detection
}

\author{
Ricardo Faria ${ }^{1}$, Henrique Vicente ${ }^{2}$, António Abelha ${ }^{3}$, Manuel Santos ${ }^{4}$, \\ José Machado ${ }^{3}$, and José Neves ${ }^{3(\bowtie)}$ \\ 1 Departamento de Informática, Universidade do Minho, Braga, Portugal \\ ricardo.mof@hotmail.com \\ 2 Departamento de Quimica, Escola de Ciências e Tecnologia, \\ Universidade de Évora, Évora, Portugal \\ hvicente@uevora.pt \\ 3 Centro ALGORITMI, Universidade do Minho, Braga, Portugal \\ \{abelha, jmac, jneves\}@di. uminho.pt \\ 4 Centro ALGORITMI, Universidade do Minho, Guimarães, Portugal \\ mfs@dsi.uminho.pt \\ http://algoritmi.uminho.pt
}

\begin{abstract}
The nosocomial infections are a growing concern because they affect a large number of people and they increase the admission time in healthcare facilities. Additionally, its diagnosis is very tricky, requiring multiple medical exams. So, this work is focused on the development of a clinical decision support system to prevent these events from happening. The proposed solution is unique once it caters for the explicit treatment of incomplete, unknown, or even contradictory information under a logic programming basis, that to our knowledge is something that happens for the first time.
\end{abstract}

Keywords: Nosocomial infection · Healthcare $\cdot$ Knowledge representation and reasoning - Logic programming - Case-based reasoning • Similarity analysis 